\title{
Pluripotency and differentiation of embryonic stem cells ${ }^{*}$
}

\author{
Yinyin Liu ${ }^{1}$, Haibo Zhao ${ }^{1}$, Liang Liang ${ }^{1}$, Peilei Fan ${ }^{1}$, Yujia Zhao ${ }^{1}$, Jinling Feng ${ }^{1}$, Ying Zhang ${ }^{1}$, Yang Gao ${ }^{1}$ and Zhengsheng \\ Shen $^{1 * *}$ \\ ${ }^{1}$ Beijing Institute of Metrology, Beijing, 100024, China \\ ** Corresponding author
}

\begin{abstract}
Mouse embryonic stem (ES) cells derive from the inner cell mass of an early embryo called blastocyst, making them promising resource for regenerative medicine. They possess two unique properties: self-renewal and pluripotency. Different ways can be used to assess which extracellular signal and factor inside ES cells has an impact on the pluripotency of ES cells. Nowadays, many extracellular signals and transcription factors have been identified, such as extracellular signals like LIF and transcription factors like Oct4. Studying the mechanism and function of these factors offers great insight and advance our understanding of pluripotency and self-renewal and thus shed light on regenerative medicine.
\end{abstract}

\section{Origins and properties of embryonic stem cells}

Mouse embryonic stem (ES) cells are cells derived from the inner cell mass of an early embryo called blastocyst by isolating and cultivating blastocyst on mouse embryonic fibroblast (MEF) layer or with embryonic carcinoma cell conditioned medium followed by expanding the primary cell outgrowth. The culture of mouse ES cells in vitro was first achieved by two groups independently in 1981',2 (Evans and Kaufman, 1981; Martin, 1981). These cells have two unique properties: self-renewal and pluripotency, which means they can divide indefinitely or differentiate into all cell types of body under proper condition ${ }^{3-5}$.

\section{Approaches to test pluripotency}

There are different ways to assess the pluripotency in vitro and in vivo (Figure 1). From the least stringent way to the most stringent way are expression of pluripotency markers, in vitro differentiation essay, teratoma formation, chimera and germline transmission, and tetraploid complementation.

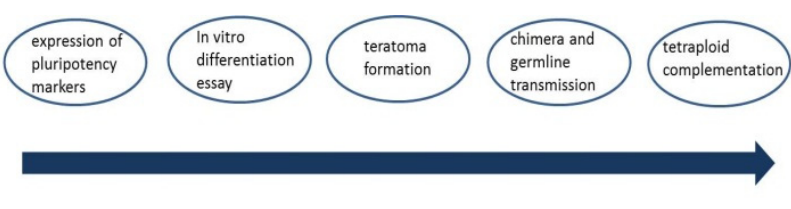

least stringent

most stringent

Figure 1 multiple ways to assess the pluripotency of ES cells

\subsection{In vitro differentiation}

Embryoid body (EB) formation is one of the commonly used methods to differentiate ES cells in vitro. In this method, ES cells are grown in the absence of LIF in hanging drops on the culture dish and form threedimensional aggregates called EB. EB formed by pluripotent cells consists of all three germ layers, ectoderm, endoderm and mesoderm and recapitulates many aspects of cell differentiation during early mammalian embryogenesis.

\subsection{Teratoma formation}

Teratoma formation is an approach to test pluripotency more in vivo, compared to EB formation. In this method, single cell suspension is injected into the leg of nude mice. Pluripotent cells will grow into tumors called teratoma and differentiate into all three germ layers.

\subsection{Chimera and germline contribution}

Chimera and germline contribution is to examine whether the in vitro cultured pluripotent cells can contribute to normal animal development and transmission into offspring following injection into blastocysts. In contrast to teratoma formation, the tested pluripotent cells are able to integrate into normal tissues, serve their functions and even transmit to the next generation.

\subsection{Tetraploid complementation}

Tetraploid complementation assay, which is considered the most stringent way to test pluripotency so far, shows that pluripotent cells alone are capable of supporting 
embryonic development. In this method, a tetraploid cell is generated through fusion of two blastomeres from two-cell stage embryos. Through combination of tetraploid blastocysts with diploid pluripotent cells, the diploid cells give rise to embryos due to complementation while the tetraploid cells only contribute to extraembryonic tissues. So fetuses and viable offspring are completely derived from pluripotent cells.

\section{Extracellular signals}

Extracellular signals have been found to play an important role in the maintenance of ES cell pluripotency or stimulating differentiation down defined lineages 6,7 .

\subsection{Signals that contribute to maintenance of pluripotency}

It's known that mouse ES cells depend on LIF to maintain their self-renewal in the regular cell culture ${ }^{6}$ while LIF is not required for pluripotency in vivo ${ }^{8}$. After LIF binds to its receptor, it activates three parallel pathways and eventually leads to the expression of Oct4, Nanog and Sox $2^{9,10,11}$. It's known Nanog can promote self-renewal of mouse ES cells in the absence of LIF ${ }^{12}$. Besides Nanog, only limited genes can promote selfrenewal when ovexpressed. Previous study showed that when Klf4, Tbx3 or Ronin is overexpressed, mouse ES cells can self-renew even in the absence of $\mathrm{LIF}^{13}$.

However, LIF itself is not adequate to maintain selfrenewal of mouse ES cells. Suppression of differentiation and concomitant efficient self-renewal requires additional signal--BMPs. After BMP binds to its receptor, it induces expression of Id proteins via Smad pathway and inhibits differentiation and sustains selfrenewal in collaboration with STAT3 ${ }^{14}$.

Other factors, such as Wnt, activin/nodal, and bFGF, have also been shown to contribute to maintenance of pluripotency, at least under certain culture conditions. Moreover, human ES cells and the human fibroblasts as their feeder cells have been reported to send reciprocal signals of FGF and IGF, respectively, sufficient to maintain the pluripotency of the ES cells ${ }^{15}$.

\subsection{Signals that promote differentiation}

Retinoic acid, a particularly well-characterized inducer of differentiation, has been shown to induce neural differentiation of mouse embryonic stem cells and directly contribute to silencing of the Oct4 locus. Notch is another factor to promote neural differentiation in both human and mouse embryonic stem cells ${ }^{16}$.

\section{Master transcription factors for pluripotency in ES cells}

It's generally accepted that Oct4, Nanog and Sox 2 are the most important pluripotent factors in ES cells, and induced pluripent stem cells. However, only Oct4 is required for the pluripotency and self-renewal of ES cells while both Sox 2 and Nanog are not required for the maintenance of ES cells. All of them are required for embryogenesis except Sox2, which is possibly due to the presence of maternally derived Sox 2 protein. Moreover, among these three factors only Nanog can promote selfrenewal of mouse ES cells in the absence of $\mathrm{LIF}^{17}$.

\subsection{Oct4}

OCT4 is a homeodomain TF of the POU family and belongs to the Octamer class that recognizes the 8-bp DNA sequence ATGCAAAT. A common feature of the POU family of TFs is the presence of a highly conserved motif, the POU domain, which consists of two structurally independent subdomains: a 75-amino acid POU specific (POUs) region at the N-terminus and a 60 amino-acid homeodomain (POUh) at the C-terminus. Both subdomains make specific contact with DNA through a helix-turn-helix structure and are separated by a variable linker of 15 to 56 amino-acids. Regions outside the POU domain, the N-terminal $(\mathrm{N})$ domain and the C-terminal (C) domain, are not critical for DNA binding and exhibit little sequence conservation. Both the $\mathrm{N}$ domain and the $\mathrm{C}$ domain play important roles in transactivation. Oct4 is expressed as early as in the unfertilized egg and subsequently distributed to all cells until blastocyst is formed, when Oct4 expression is gradually diminished in the trophoblast and confined to all cells of epiblast. The expression of OCT4 is downregulated during ES cell differentiation. Repression of OCT4 results in the differentiation of ES cells into trophectoderm and in vitro and differentiation of epiblast (pluripotent cells) in vivo ${ }^{18}$.

\subsection{Nanog}

Nanog was identified by a screen where overexpression of Nanog allows mouse ES cells to self-renew after LIF withdrawal. It's named after Tir nan $\mathrm{Og}$, the land of ever young. During embryogenesis, its expression starts at the morula stage. It's predominantly expressed in pluripotent cells, including ICM, epiblast, primordial germ cells, ES cells and EG cells. Nanog is an NK-2 calss homeobox transcription factor. It contains a homeodomain responsible for DNA binding and tryptophan repeats motif involved in protein-protein interaction. The DNA sequence recognized by Nanog is still controversial. Systematic evolution of ligands by exponential enrichment (SELEX) revealed that Nanog binds to the core homeodomain recognition sequence TAAT while crystal structure analysis of its homeodomain alone suggests that Nanog targets TAATGG. However, a genome-wide mapping study based on chromatin immunoprecipitation found Nanog recognize the sequence CATT, which is defferent from in vitro derived described above. One possible explanation for such difference is that localization mapping by ChIP more reflects in vivo binding sites for transcription factors, 
which may results from cooperative binding by multiple associated proteins that also have affinity for chromatin ${ }^{19}$.

\subsection{Sox2}

Sox 2 belongs to the Sry-related HMG box (Sox) DNAbinding-protein family. It is expressed not only in pluripotent cells including ICM, epiblast, and germ cells but also multipotent cells such as extraembryonic ectoderm and neural stem cells. Decreased level of Sox2 in ES cells by RNAi knockdown induces differentiation into trophectoderm. It's involved in the regulation of transcription and chromatin structure. Oct4 and Sox2 often act synergistically to bind to adjacent DNA sequences and activate gene transcription. Although Oct4/DNA crystal structure has not been reported, Oct1/Sox2/Hoxb1-DNA ternary complex and POU/HMG/DNA ternary complex suggest that the HMG domain of Sox 2 and POU domain of Oct 4 might interact with each other and position the POU domain to bind to DNA $^{20}$

\section{The core transcriptional circuitry influencing pluripotenecy}

Both proteomic and genomic studies have helped illustrate the network of proteins that interact with Nanog and Oct4 mediated signaling pathways and contributed to our understanding of their function. These three transcription factors generate a pluripotent state of ES cells by both occupying active genes necessary to maintain self-renewal and pluripotent state of ES cells and silent genes that promote differentiation of ES cells. Moreover, they occupy each other's promoter and form a feedback and feedforward loop. Oct4, Nanog and Sox 2 also participate directly or indirectly in recruiting chromatin modification enzymes and chromatin remodeling proteins to regulate gene expression.

Nanog, Sox 2 and Oct4 also generate a pluripotent state of ES cells by co-occupying a large number of target genes. In human ES cells at least 353 genes were occupied by these three factors together. About half of the promoter regions occupied by OCT4 were also bound by $\mathrm{SOX} 2$, while most of the promoter regions bound by both OCT4 and SOX2 were also occupied by NANOG. The binding sites for these factors on a single gene are often overlapping.

They co-occupy active genes necessary to maintain self-renewal and pluripotent state of ES cells and silent genes. These silent genes are mainly developmental transcription factors and expression of these genes often results in differentiation of ES cells. Moreover, they occupy each other's promoter and form a feedback and feed forward loop to maintain their own expression. For example, Kuroda's group showed that Oct4 and Sox2 together could bind to the Nanog promoter and activate its transcription. Okumura-Nakanishi and his colleagues found that Oct 4 and Sox 2 could also occupy and regulate Oct4. Masui and colleagues demonstrated that Sox2 could work together with Oct4 to activate a number of genes including Nanog, Oct4 and Sox 2 in vitro and in vivo by utilizing inducible Sox 2 deletion ES cells ${ }^{21}$.

\section{Conclusions}

Both extracellular signals and internal transcription factors contribute to the unique properties of EC cells. Understanding how these signals and transcription factors orchestrate the program necessary for maintenance of ES cells is crucial for the EC cell biology and regenerate medicine. Prospectively, the knowledge about the molecular basis of EC cell identity may help us develop better strategies to direct differentiation into desired populations of cells for therapeutic purposes.

\section{*Acknowledgements}

This work was supported by the Foundation of China (Grants No. 2019YFF0216703, KJ2019-23, 2019MK001 and Z201100009319003).

\section{References}

1. Evans, M.J., and Kaufman, M.H. (1981). Establishment in culture of pluripotential cells from mouse embryos. Nature 292, 154-156.

2. Martin, G.R. (1981). Isolation of a pluripotent cell line from early mouse embryos cultured in medium conditioned by teratocarcinoma stem cells. Proc Natl Acad Sci U S A 78, 7634-7638.

3. Jihoon Kim, Bon-Kyoung Koo, Juergen A Knoblich. (2020). Human organoids: model systems for human biology and medicine. Nat Rev Mol Cell Biol, 7, 114

4. Rossant, J. (2001). Stem cells from the Mammalian blastocyst. Stem Cells 19, 477-482.

5. Rossant, J. (2007). Stem cells and lineage development in the mammalian blastocyst. Reprod Fertil Dev 19, 111-118.

6. Burdon, T., Smith, A., and Savatier, P. (2002). Signalling, cell cycle and pluripotency in embryonic stem cells. Trends Cell Biol 12, 432-438.

7. Chen, X., Xu, H., Yuan, P., Fang, F., Huss, M., Vega, V.B., Wong, E., Orlov, Y.L., Zhang, W., Jiang, J., et al. (2008). Integration of external signaling pathways with the core transcriptional network in embryonic stem cells. Cell 133, 11061117.

8. Smith, A.G., Heath, J.K., Donaldson, D.D., Wong, G.G., Moreau, J., Stahl, M., and Rogers, D. (1988). Inhibition of pluripotential embryonic stem cell differentiation by purified polypeptides. Nature 336, 688-690.

9. Matsuda, T., Nakamura, T., Nakao, K., Arai, T., Katsuki, M., Heike, T., and Yokota, T. (1999). STAT3 activation is sufficient to maintain an undifferentiated state of mouse embryonic stem cells. EMBO J 18, 4261-4269. 
10. Niwa, H., Burdon, T., Chambers, I., and Smith, A. (1998). Self-renewal of pluripotent embryonic stem cells is mediated via activation of STAT3. Genes Dev 12, 2048-2060.

11. Niwa, H., Ogawa, K., Shimosato, D., and Adachi, K. (2009). A parallel circuit of LIF signalling pathways maintains pluripotency of mouse ES cells. Nature 460, 118-122.

12. Chambers, I., Colby, D., Robertson, M., Nichols, J., Lee, S., Tweedie, S., and Smith, A. (2003)

13. Dejosez, M., Levine, S.S., Frampton, G.M., Whyte, W.A., Stratton, S.A., Barton, M.C., Gunaratne, P.H., Young, R.A., and Zwaka, T.P. (2010). Ronin/Hcf-1 binds to a hyperconserved enhancer element and regulates genes involved in the growth of embryonic stem cells. Genes Dev 24, 1479-1484.

14. Ying, Q.L., Nichols, J., Chambers, I., and Smith, A. (2003). BMP induction of Id proteins suppresses differentiation and sustains embryonic stem cell self-renewal in collaboration with STAT3. Cell 115, 281-292.

15. Bendall, S.C., Stewart, M.H., Menendez, P., George, D., Vijayaragavan, K., Werbowetski-Ogilvie, T., Ramos-Mejia, V., Rouleau, A., Yang, J., Bosse, M., et al. (2007). IGF and FGF cooperatively establish the regulatory stem cell niche of pluripotent human cells in vitro. Nature 448, 1015-1021.

16. Chambers, I., and Smith, A. (2004). Self-renewal of teratocarcinoma and embryonic stem cells. Oncogene 23, 7150-7160.

17. Silva, J., Nichols, J., Theunissen, T.W., Guo, G., van Oosten, A.L., Barrandon, O., Wray, J., Yamanaka, S., Chambers, I., and Smith, A. (2009). Nanog is the gateway to the pluripotent ground state. Cell 138, 722-737

18. Remenyi, A., Lins, K., Nissen, L.J., Reinbold, R., Scholer, H.R., and Wilmanns, M. (2003). Crystal structure of a POU/HMG/DNA ternary complex suggests differential assembly of Oct4 and Sox 2 on two enhancers. Genes Dev 17, 2048-2059.

19. Jauch, R., Ng, C.K., Saikatendu, K.S., Stevens, R.C., and Kolatkar, P.R. (2008). Crystal structure and DNA binding of the homeodomain of the stem cell transcription factor Nanog. J Mol Biol 376, 758-770.

20. Li, J., Pan, G., Cui, K., Liu, Y., Xu, S., and Pei, D. (2007). A dominant-negative form of mouse SOX2 induces trophectoderm differentiation and progressive polyploidy in mouse embryonic stem cells. J Biol Chem 282, 19481-19492.

21. Orkin, S.H., and Hochedlinger, K. (2011). Chromatin connections to pluripotency and cellular reprogramming. Cell 145, 835-850. 